\title{
The Vorton Method
}

\section{Theory and Applications to Fluid Mechanics}

\author{
A.J.Q. ALKEMADE and F.T.M. NIEUWSTADT \\ Laboratory for Aero- and Hydrodynamics \\ Delft University of Technology, 2628 AL Delft, The Netherlands \\ and \\ E. VAN GROESEN \\ Faculty of Applied Mathematics \\ University of Twente, 7500 AE Enschede, The Netherlands
}

\begin{abstract}
After a general introduction to the vorton method, which is a vortex method resembling the 2-D point-vortex method, a set of equations describing dynamics of 3-D vortex singularities (vortons) is derived, avoiding the inconsistency in the derivation of other vorton equations which have been applied. Though inviscid, numerical simulations show reconnection phenomena.
\end{abstract}

Key words: vortex methods - vorton method - reconnection

\section{Introduction}

Application of vorticity theory to the study of turbulence is not only formally attractive. It can be claimed that the problem of turbulence is a problem of vorticity dynamics. Therefore, it seems mathematically attractive and physically acceptable to consider (turbulent) fluid dynamics in terms of "parcels" of vorticity which induce displacement and deformation on each other. This is the basic idea behind the use of vortex methods in fluid dynamics.

The vorticity vector $\boldsymbol{w}$ is defined by:

$$
\boldsymbol{w} \equiv \boldsymbol{\nabla} \times \boldsymbol{v}
$$

where $\boldsymbol{v}$ is the velocity field.

3-D inviscid vortex deformation (reorientation and stretching) is described by the Helmholtz equation:

$$
\frac{D w_{i}}{D t}=S_{i j} w_{j}
$$

where the rate of strain tensor $S_{i j} \equiv \frac{1}{2}\left(\frac{\partial v_{i}}{\partial x_{j}}+\frac{\partial v_{j}}{\partial x_{i}}\right)$.

\section{The Vorton Method}

The vorton method can shortly be described by: 
- The "vortex elements" are, essentially, 3-D point vortices or coreless infinitesimal vortex "blobs".

- The method is completely local/Lagrangian: the deformation of the vortons is followed on their paths through 3-D space.

- Displacement and deformation of a vorton are derived from the vorton velocity field caused by all the other vortons. Deformation is derived from the Helmholtz equation (2).

A vorton labelled $\alpha$ has a location vector, $\boldsymbol{r}_{\alpha}$, and a vorticity intensity vector, $\boldsymbol{\gamma}_{\alpha}$. A vorton is represented by an arrow.

For every vorton we need a set of displacement and deformation equations, which can be derived once we have found the vorton velocity and vorticity field. These fields can be found from the following vorton vector-potential field:

$$
\boldsymbol{A}(\boldsymbol{x}, t)=\frac{1}{4 \pi} \sum_{\alpha} \frac{\boldsymbol{\gamma}_{\alpha}}{R_{\alpha}} \equiv \sum_{\alpha} \phi_{\alpha}(\boldsymbol{x}, t) \boldsymbol{\gamma}_{\alpha}(t)
$$

where $R_{\alpha} \equiv\left|\boldsymbol{x}-\boldsymbol{x}_{\boldsymbol{\alpha}}\right|$.

From this vector-potential field we find the following velocity field:

$$
\boldsymbol{v}(\boldsymbol{x}, t)=\boldsymbol{\nabla} \times \boldsymbol{A}=\sum_{\alpha} \boldsymbol{\nabla} \phi_{\alpha} \times \boldsymbol{\gamma}_{\alpha}
$$

Finally, from (3) we get the following vorticity field by applying (1):

$$
\begin{aligned}
\boldsymbol{w}(\boldsymbol{x}, t) & =\sum_{\alpha}\left\{\boldsymbol{\gamma}_{\alpha} \delta\left(\boldsymbol{R}_{\alpha}\right)+\left(\phi_{\alpha}^{\prime \prime}\right) \circ \boldsymbol{\gamma}_{\alpha}\right\} \\
& =\sum_{\alpha}\left\{\boldsymbol{\gamma}_{\alpha} \delta\left(\boldsymbol{R}_{\alpha}\right)-\boldsymbol{\nabla}\left[\boldsymbol{\nabla} \cdot\left(\phi_{\alpha} \boldsymbol{\gamma}_{\alpha}\right)\right]\right\}
\end{aligned}
$$

Here $\left(\phi_{\alpha}^{\prime \prime}\right)$ is a matrix whose $i j$-th element is given by $\partial^{2} \phi_{\alpha} / \partial x_{j} \partial x_{i}$.

If we compare this field with the non-divergencefree vorton vorticity field originally introduced by Novikov in [5] (and also used by Kuwabara in [4]), which consists of the delta-functions only, we conclude that the second (gradient) part of (4) makes the vorticity field divergencefree. It can be regarded as the nonlocal vorticity field surrounding the original singular vorton represented by the first part. So vortons are not really $3-\mathrm{D}$ point vortices.

In order to avoid (infinite) "self-displacement", the displacement equation of vorton $\alpha$ is derived by applying the velocity field $\tilde{\boldsymbol{v}}_{\alpha}$ induced by all other vortons $\beta \neq \alpha$ :

$$
\dot{\boldsymbol{r}}_{\alpha}=-\sum_{\beta \neq \alpha} \boldsymbol{\gamma}_{\beta} \times\left.\left(\boldsymbol{\nabla} \phi_{\beta}\right)\right|_{\boldsymbol{x}=\boldsymbol{r}_{\alpha}}
$$

The deformation equation for vorton $\alpha$ is derived from eq. (2) by substituting velocity field $\tilde{\boldsymbol{v}}_{\alpha}$ and substituting $\boldsymbol{\gamma}_{\alpha}$ for $\boldsymbol{w}$. 
After elaboration we get:

$$
\dot{\gamma}_{\alpha}=\frac{1}{2}\left\{N_{\alpha}+K_{\alpha}\right\}
$$

where

$$
N_{\alpha} \equiv \sum_{\beta \neq \alpha}\left[\left(\phi_{\alpha \beta}^{\prime \prime}\right) \circ \boldsymbol{\gamma}_{\alpha}\right] \times \boldsymbol{\gamma}_{\beta}
$$

and

$$
K_{\alpha} \equiv \sum_{\beta \neq \alpha}\left(\phi_{\alpha \beta}^{\prime \prime}\right) \circ\left(\boldsymbol{\gamma}_{\beta} \times \boldsymbol{\gamma}_{\alpha}\right)
$$

The original equations used by Novikov in [5] are equal to our expression $N_{\alpha}$. The equations used by Kuwabara in [4] are equal to our expression $K_{\alpha}$. It can be shown that only our formulation (6) is consistent with the vorticity field (4).

\section{Requirements on the vorton method}

Certain requirements should be imposed on vortex methods in general. We will treat them here for the vorton method:

- Most important, from a physical point of view, are criteria for the "physical reality" of the method.

One of the fundamental aspects of vortex dynamics is vortex reconnection.

An interesting simulation in this context is that of the behaviour of a pseudoelliptical vorton ring. In contrast to the vorton ring, it indces its own deformation. The kind of deformation is dependent of its aspect ratio. The first results obtained by one of the authors (FA) show resemblance to those of earlier simulations by others [3]; see fig. 1 .

Simulations like these may show the specific characteristics of the several vorton equations which have been proposed. However, strong criteria for "correct" reconnection behaviour do not seem to exist.

- The discretization by finitely many elements should converge to the exact smooth solutions as the number of elements goes to infinity.

For the relative simple case of the vortex ring, this has been shown analytically by Novikov [5]. A definitive general proof has been given in [2].

- The method should not require a disproportionate amount of computational effort and computation time.

So far our numerical simulations could be performed on an ordinary minicomputer (HP9000); computational times were of order of one hour.

Though at present only the subject of wishful thinking, eventually the vorton method may be applied to study turbulent boundary layer flows. A first attempt was shown in [1]: a vorton ring in a shear flow above a flat plate transformed into a horseshoe vortex. Such simulations are being performed by the authors at this moment. 


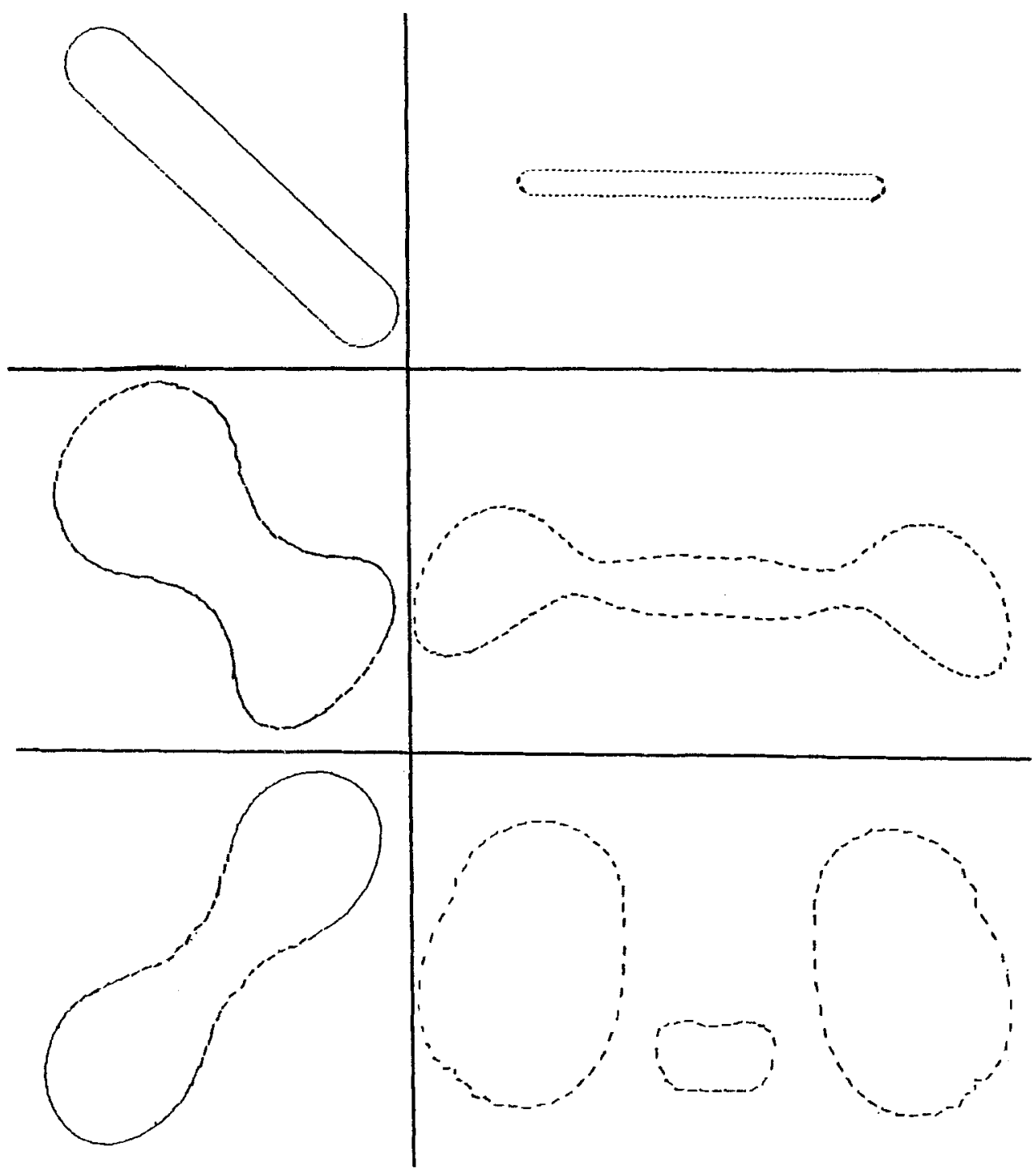

Fig. 1. The deformation of a pseudo-elliptical vorton ring in time for relatively small (left) and large (right) aspect ratio. In the former case, only "switching" of the ring occurs, in the latter case reconnection occurs and the ring breaks up. 


\section{Acknowledgements}

The authors thank Gianni Pedrizetti who kindly provided his numerical code on which ours has been based. The figures in this paper were produced by Roy DeJong and Dirkjan van Dam.

\section{References}

1. M.I. Aksman, E.A. Novikov: 1988, "Reconnections of vortex filaments". Fluid Dyn. Res. 3, 239.

2. T.Y. Hou, J. Lowengrub: 1990, "Convergence of the point vortex method for the 3-D Euler equations". Comm. Pure Appl. Math. 43, 965.

3. M. Kiya, H. Ishii: 1991, "Deformation and splitting of pseudo-elliptical vortex rings". In: $A d$ vances in Turbulence 3 (eds. A. $Y$. Johanson, P.H. Alfredsson). Springer, Heidelberg.

4. S. Kuwabara: 1988, "Pseudo-canonical formulation of 3-dimensional vortex motion and vorton model analysis". Fluid Dyn. Res. 3, 163.

5. E.A. Novikov: 1983 , "Generalized dynamics of three-dimensional vortical singularities (vortons)". Sov. Phys. JETP 57, 566. 